\title{
The Policy Advice on the G4B Enhancement - Deducting Policy Priorities through AHP Analysis ${ }^{1}$ -
}

\author{
ChoongSik Chung ${ }^{1}$, Wookjoon Sung ${ }^{2}$ \\ ${ }^{1}$ Dept. of Public Administration, Kyungsung University \\ 314-79. daeyeon-dong, nam-gu, Busan 608-736 Korea \\ cschung@ks.ac.kr \\ ${ }^{2}$ Graduate School of Public Policy \& Infomation Tech., Seoul National University \\ of Science \& Technology \\ 232 Gongneung-ro, Nowon-gu, Seoul, 139-743, Korea \\ Corresponding Author: wjsung@seoultech.ac.kr
}

\begin{abstract}
The object of this research is to outline policy priorities through AHP (Analytic Hierarchy Process) analysis that can upgrade the online service systems for business. This study categorized policy factors for G4B development into three different groups, such as system improvement, service improvement, and policy improvement. The results showed that "G4B policy improvement" was considered the most important assignment, while "G4B service improvement" was considered to have the highest priority in terms of urgency. From the result of analysis, this study proposed several policies such as the development of new services, a link between similar business platforms, the policy coordination, and the reorganization of the promotion system.
\end{abstract}

Keywords: Government for Business (G4B), Creative Economy, Electronic Government, Electronic Service Delivery

\section{Introduction}

Through the development of its information and communication industry, Korea has been placed in first position in the UN Electronic Government (e-government) rankings in 2010, 2012, and 2014. Korea is also constantly expanding its e-government online services such as G4C, G2B, and G4B. In particular, with the launch of the GeunHye Park administration, government 3.0 has been introduced under the banner of openness, sharing, collaboration, and a creative economy that ties the creative ideas of citizens and businesses into new values has been emphasized as the main driving force behind the development of the nation [1-3].

Today, it is impossible to secure the competitive power of businesses without the improvement of a society's economic power. Although Korea is globally competitive in business and is developing its information and communication industry, governmental support for the utilization of this competitiveness and development and efforts to construct ecological environments have been lacking. In particular, electronic civil affairs administration services must be upgraded so e-government systems can be implemented and the citizens can see the effect of implementing this form of governance. In addition to this, the necessity of providing an efficient business environment and effective administration support system for businesses is highlighted [4].

1 This work was supported by the National Research Foundation of Korea Grant funded by the Korean Government (NRF-2014S1A3A2044645) 
The objective of this research is to outline policy priorities through AHP (Analytic Hierarchy Process) analysis and propose improvements that can upgrade the online service systems for businesses. Especially, this is reviewed based on Government for Business (G4B), which is designed to make the business civil complaint service more efficient in implementing online business support activities. AHP (Analytic Hierarchy Process) is used as a research method to ascertain priorities for policy alternatives.

\section{Definitions of G4B and Its Status}

If the civil complaints 24 (G4C) system in the electronic civil affairs service is an allcitizen service, G4B is the service for business. G4B began with the Roh administration's creation of Government to Business (G2B) [5]. Then, the Lee administration selected G4B as the "main assignment for the new generation of e-government" in order to constantly promote upgrading the system.

G4B aims to provide an electronic governmental portal service for businesses using the world-class ICT infrastructure of the country. Specifically, it intends to implement Single Gateway as a means of supporting business competitiveness and focusing on the services where businesses are highly competitive. This is achieved by linking business-supporting ICT infrastructures distributed to multiple institutions such as governmental/public facilities. The current G4B sites provide services for an integrated guiding and registering of businesses' civil complaints, the modification of overall business content, an integrated registering and issuing of exams/verification/record, registering of business bottlenecks, financial aid information, consultation support, etc. (http://www.g4b.go.kr). However, it is still difficult to find an academic evaluation of such business-supporting Single Gateways [6]. At this point, performance reviews, political analysis, and proposals for better services are vital.

\section{Drawing Policy Priorities through AHP Analysis}

\subsection{Research Design}

In this research, AHP (Analytic Hierarchy Process) methodologies were applied in order to deduct policy priorities for the constant use and spread of G4B services. AHP is a multi-standard decision-making method developed by Saaty and which originated from OR (Operations Research). It has been used for various research purposes such as planning, resource distributions, conflict resolutions, and predictions [7].

The AHP analysis method applies the participants' subjective decisions by utilizing pairwise comparisons between the variables and quantifying their decisions. Therefore, this method is effective when proposing a policy [8, 9]. KDI has been using AHP to review the preliminary validity of all national projects with an investment of 50 billion Won or more since 1999 [10].

\subsubsection{Hierarchical Classification of Policy Factors for the Development of G4B Services}

In this research, relevant literature was reviewed in order to categorize G4B policy factors systematically. For this, we analyzed research that evaluated G2B and G4B [1116]. Additionally, literature related to the common utilization of Civil Complaints 24, which is considered to be the most successful multi-departmental electronic government business in Korea, and administrative information was reviewed [1, 17-20]. We also referred to the analysis and classification of research related to e-government business evaluation methodologies $[12,21]$

Based on a literature review, we categorized policy factors for G4B development into three different groups, such as system improvement, service improvement, and policy 
improvement, and created a development policy index and factors for each group. Each factor was reviewed in terms of importance and urgency. The importance indicates the key policy while urgency indicates the policy and improvements that must be promptly made and supported along with other improvements in order to facilitate the spread of G4B site application.

The following are the development policy indexes and factors for each area. First, system improvement consists of constant system maintenance (hardware/SW/UI improvement, linkage of the relevant system), security issue improvement (authentication of SW improvement, personal information password improvement), etc., Second, the service improvement area consists of online administration service improvement (expansion of business complaints services and support, customized services for the business cycle) and business support based on big data (providing public/private DATA SET and service linkage, provide consulting and information for creating businesses). Third, the method improvement area consists of legal policy improvement (providing legal evidence for linkage/integration service, establishing promotion strategies for G4B service and roadmap) and promotional system improvement (constructing collaborative systems between sub departments, establishing specific organizations, and creating interministerial adjustment organizations). The explanation and systematic chart for the development policy factors are shown in Table 1.

Table 1.

\begin{tabular}{|c|c|c|c|}
\hline Major Class & Mid Class & Minor Class & Main Content \\
\hline \multirow{4}{*}{$\begin{array}{c}\text { G4B } \\
\text { System } \\
\text { Improvement }\end{array}$} & \multirow{2}{*}{ Maintain and Repair System } & $\begin{array}{l}\text { Hardware/SW/UI } \\
\text { Improvement }\end{array}$ & $\begin{array}{c}\text { G4B Site Internal System Improvement } \\
\text { (Business Support Hub Construction, etc.) and } \\
\text { User Convenience Support }\end{array}$ \\
\hline & & $\begin{array}{l}\text { Linking the Relevant } \\
\text { Systems }\end{array}$ & $\begin{array}{l}\text { Construct a Linked System with Business- } \\
\text { support-relations with other sites (Civil } \\
\text { Complaint 24, Business Garden, etc.) }\end{array}$ \\
\hline & \multirow[b]{2}{*}{ Security Issue Improvement } & $\begin{array}{l}\text { Public Authentication SW } \\
\text { Improvement }\end{array}$ & Enhance the Security of the G4B System \\
\hline & & $\begin{array}{c}\text { Personal Member } \\
\text { Information Encryption } \\
\text { Improvement }\end{array}$ & $\begin{array}{c}\text { Enhance G4B Users' Personal Information } \\
\text { Protection }\end{array}$ \\
\hline \multirow{4}{*}{$\begin{array}{c}\text { G4B } \\
\text { Service } \\
\text { Improvement }\end{array}$} & \multirow{2}{*}{$\begin{array}{l}\text { Online Administration } \\
\text { Service } \\
\text { Improvement/Expansion }\end{array}$} & $\begin{array}{l}\text { Business Civil Affairs } \\
\text { Service and Support } \\
\text { Expansion }\end{array}$ & $\begin{array}{c}\text { Expand the Range of Provided Services for } \\
\text { Current Business Content's Overall Changes, } \\
\text { Business Errors, Issue } \\
\text { Test/Authentication/Record, Business } \\
\text { Complaints }\end{array}$ \\
\hline & & $\begin{array}{l}\text { Provide Customized Services } \\
\quad \text { for Business Lifecycles }\end{array}$ & $\begin{array}{c}\text { Implement One-stop Service for Each Business } \\
\text { Type (Small Merchant, Small Business, } \\
\text { Manufacturing, etc.) and for Each Business } \\
\text { Process (Start, Manage, Change, Close } \\
\text { Business, etc.) }\end{array}$ \\
\hline & \multirow{2}{*}{$\begin{array}{l}\text { Big Data-based Business } \\
\text { Extraction Support }\end{array}$} & $\begin{array}{l}\text { Provide Public/Private DATA } \\
\text { SET and Service Linkage }\end{array}$ & $\begin{array}{l}\text { Provide business items through a mesh-up of } \\
\text { releasing public and private data }\end{array}$ \\
\hline & & $\begin{array}{l}\text { Provide Consulting and } \\
\text { Information for Business } \\
\text { Extraction }\end{array}$ & $\begin{array}{l}\text { Provide consultation and business models (BM) } \\
\text { for the commercialization of ideas }\end{array}$ \\
\hline \multirow{3}{*}{$\begin{array}{c}\text { G4B } \\
\text { Policy } \\
\text { Improvement }\end{array}$} & \multirow[t]{2}{*}{ Legal policy Improvement } & $\begin{array}{l}\text { Find Legal Evidence of } \\
\text { Linked/Integrated Service }\end{array}$ & $\begin{array}{l}\text { Support linking \&sharing information between } \\
\text { departments, special policies for ICT, review of } \\
\text { enactments and amendments of related laws } \\
\text { such as Information and Communications } \\
\text { Industry Promotion Act }\end{array}$ \\
\hline & & $\begin{array}{l}\text { Establish G4B Service } \\
\text { Promotion Strategy and } \\
\text { Roadmap }\end{array}$ & $\begin{array}{c}\text { Establish a roadmap and construct specific } \\
\text { promotion strategies for the systematic } \\
\text { development of G4B services }\end{array}$ \\
\hline & $\begin{array}{l}\text { Promoted System } \\
\text { Improvement }\end{array}$ & $\begin{array}{l}\text { Construct Collaborating } \\
\text { Systems between } \\
\text { Departments }\end{array}$ & $\begin{array}{l}\text { Construct parallel collaboration guidelines for } \\
\text { creating a discussion board for the Ministry of } \\
\text { Science, ICT, and Future Planning, Ministry of } \\
\text { Trade, Industry \& Energy, Small and Medium } \\
\text { Business Administration, Ministry of Security } \\
\text { and Public Administration, etc. }\end{array}$ \\
\hline
\end{tabular}




\begin{tabular}{||c||c||c}
\hline \multicolumn{1}{||c||}{} & $\begin{array}{c}\text { Establish Exclusive } \\
\text { Organizations }\end{array}$ & $\begin{array}{c}\text { Construct a department to manage the entire } \\
\text { on/off business support services distributed to } \\
\text { various departments }\end{array}$ \\
\cline { 2 - 3 } & $\begin{array}{c}\text { Prepare inter-ministerial } \\
\text { Adjustment Organizations }\end{array}$ & $\begin{array}{c}\text { Install high-rank adjustments to resolve the } \\
\text { current functional overlap between the currently } \\
\text { distributed business service systems }\end{array}$ \\
\hline
\end{tabular}

\subsubsection{Research Method}

In this research, online surveys were conducted on academic professionals, government workers, public institution workers, and relevant business employees between February 10, 2014 and February 14, 2014. The survey questions were distributed via email to a total of 30 participants and 28 professionals responded. 2 To resolve the consistency issue that appears in pairwise comparisons, the verification of Consistency Ratio: CR was conducted during the AHP analysis. The responses were excluded when the CR goes beyond the basic value $(\mathrm{CR}=0.2) 3$.

\section{Results of AHP Analysis and Proposal}

\subsection{Result of AHP Analysis}

\subsubsection{Analysis in Terms of Policy Importance}

In major level categories, the weighted value of G4B policy improvement was the highest at $0.439(43.9 \%)$. The weighted value of service improvement was the second at $0.428(42.8 \%)$, and the weighted value of system improvement was the lowest at $0.133(13.3 \%)$. In mid-level categories, weighted value is as follows: online administration service improvement/expansion (0.287), legal policy improvement (0.236), Promoted system improvement (0.203), big data based business extraction support $(0.141)$, maintain and repair system (0.075), and security issue improvement (0.058). <Table 2> shows the detailed result of AHP analysis in terms of policy importance.

Table 2.

\begin{tabular}{|c|c|c|c|c|c|}
\hline Major Class & $\begin{array}{l}\text { the weighted } \\
\text { value } \% \\
\text { (order) }\end{array}$ & Mid Class & $\begin{array}{l}\text { the weighted } \\
\text { value } \% \\
\text { (order) }\end{array}$ & Minor Class & $\begin{array}{l}\text { the weight of } \\
\text { importance } \\
\text { (order) }\end{array}$ \\
\hline \multirow{3}{*}{$\begin{array}{c}\text { G4B } \\
\text { System } \\
\text { Improvement }\end{array}$} & \multirow{3}{*}{$\begin{array}{l}13.3 \\
(3)\end{array}$} & \multirow{2}{*}{$\begin{array}{l}\text { Maintain and Repair } \\
\text { System }\end{array}$} & \multirow{2}{*}{$\begin{array}{l}7.5 \\
(5)\end{array}$} & $\begin{array}{l}\text { Hardware/SW/UI } \\
\text { Improvement }\end{array}$ & $\begin{array}{c}1.4 \\
(13)\end{array}$ \\
\hline & & & & $\begin{array}{l}\text { Linking the Relevant } \\
\text { Systems }\end{array}$ & $\begin{array}{l}6.0 \\
(9)\end{array}$ \\
\hline & & $\begin{array}{l}\text { Security Issue } \\
\text { Improvement }\end{array}$ & $\begin{array}{l}5.8 \\
(6)\end{array}$ & $\begin{array}{l}\text { Public Authentication } \\
\text { SW Improvement }\end{array}$ & $\begin{array}{c}2.1 \\
(12)\end{array}$ \\
\hline
\end{tabular}

2Academia has selected professors that teach management information theories and electronic government theories. For those who work at government institutions, researchers who worked at the National IT Industry Promotion Agency and Korea Information Society Development Institute and conducted G4B-related tasks and research were selected. For private businesses, employees who worked at LGCNS and Samsung SDS and whose work concerned G4B BPR were selected. For government workers, individuals who had worked with administrative information sharing groups and information combination electronic centers in the past as senior members or higher were selected.

3 In order to implement logical consistency in relation to the choice makers, social science generally uses CR $<0.2$ as the standard for reviewing the decision-making process. 


\begin{tabular}{|c|c|c|c|c|c|}
\hline & & & & $\begin{array}{l}\text { Personal Member } \\
\text { Information Encryption } \\
\text { Improvement }\end{array}$ & $\begin{array}{l}3.7 \\
(11)\end{array}$ \\
\hline \multirow{4}{*}{$\begin{array}{c}\text { G4B } \\
\text { Service } \\
\text { Improvement }\end{array}$} & \multirow{4}{*}{$\begin{array}{l}42.8 \\
(2)\end{array}$} & \multirow{2}{*}{$\begin{array}{l}\text { Online Administration } \\
\text { Service } \\
\text { Improvement/Expansion }\end{array}$} & \multirow{2}{*}{$\begin{array}{c}28.7 \\
(1)\end{array}$} & $\begin{array}{l}\text { Business Civil Affairs } \\
\text { Service and Support } \\
\text { Expansion }\end{array}$ & $\begin{array}{c}10.3 \\
(3)\end{array}$ \\
\hline & & & & $\begin{array}{l}\text { Provide Customized } \\
\text { Services for Business } \\
\text { Lifecycles }\end{array}$ & $\begin{array}{c}18.4 \\
(1)\end{array}$ \\
\hline & & \multirow{2}{*}{$\begin{array}{l}\text { Big Data-based } \\
\text { Business Extraction } \\
\text { Support }\end{array}$} & \multirow{2}{*}{$\begin{array}{l}14.1 \\
(4)\end{array}$} & $\begin{array}{c}\text { Provide Public/Private } \\
\text { DATA SET and Service } \\
\text { Linkage }\end{array}$ & $\begin{array}{l}6.5 \\
(8)\end{array}$ \\
\hline & & & & $\begin{array}{l}\text { Provide Consulting and } \\
\text { Information for Business } \\
\text { Extraction }\end{array}$ & $\begin{array}{l}7.6 \\
(5)\end{array}$ \\
\hline \multirow{5}{*}{$\begin{array}{c}\text { G4B } \\
\text { Policy } \\
\text { Improvement }\end{array}$} & \multirow{5}{*}{$\begin{array}{l}43.9 \\
(1)\end{array}$} & Legal & 23.6 & $\begin{array}{l}\text { Find Legal Evidence of } \\
\text { Linked/Integrated } \\
\text { Service }\end{array}$ & $\begin{array}{l}15.3 \\
(2)\end{array}$ \\
\hline & & policyImprovement & (2) & $\begin{array}{c}\text { Establish G4B Service } \\
\text { Promotion Strategy and } \\
\text { Roadmap }\end{array}$ & $\begin{array}{l}8.3 \\
(4)\end{array}$ \\
\hline & & \multirow{3}{*}{$\begin{array}{l}\text { Promoted System } \\
\text { Improvement }\end{array}$} & \multirow{3}{*}{$\begin{array}{c}20.3 \\
(3)\end{array}$} & $\begin{array}{l}\text { Construct Collaborating } \\
\text { Systems between } \\
\text { Departments }\end{array}$ & $6.9(7)$ \\
\hline & & & & $\begin{array}{l}\text { Establish Exclusive } \\
\text { Organizations }\end{array}$ & $\begin{array}{l}6.0 \\
(10)\end{array}$ \\
\hline & & & & $\begin{array}{c}\text { Prepare inter-ministerial } \\
\text { Adjustment } \\
\text { Organizations }\end{array}$ & $\begin{array}{l}7.4 \\
(6)\end{array}$ \\
\hline total & $100 \%$ & total & $100 \%$ & total & $100 \%$ \\
\hline
\end{tabular}

\subsubsection{Analysis in Terms of Policy Urgency}

In the "major class" categories, the weighted value of G4B service improvement was the highest at $0.476(47.6 \%)$. The weighted value of policy improvement was the second at $0.372(37.2 \%)$, and the weighted value of system improvement was the lowest at $0.151(15.1 \%)$. In mid-level categories, weighted value is as follows: online administration service improvement/expansion (0.331), Promoted system improvement (0.209), legal policy improvement $(0.164)$, big data based business extraction support (0.145), maintain and repair system (0.085), and security issue improvement (0.066). <Table $3>$ shows the detailed result of AHP analysis in terms of policy importance.

Table 3.

\begin{tabular}{|c|c|c|c|c|c|}
\hline Major Class & $\begin{array}{c}\text { the weighted } \\
\text { value } \% \\
\text { (order) }\end{array}$ & Mid Class & $\begin{array}{c}\text { the weighted } \\
\text { value } \% \\
\text { (order) }\end{array}$ & Minor Class & $\begin{array}{c}\text { the weight of } \\
\text { urgency } \\
\text { (order) }\end{array}$ \\
\hline \multirow{3}{*}{$\begin{array}{c}\text { G4B } \\
\text { System } \\
\text { Improvement }\end{array}$} & \multirow{3}{*}{$\begin{array}{c}15.1 \\
(3)\end{array}$} & \multirow{2}{*}{$\begin{array}{l}\text { Maintain and Repair } \\
\text { System }\end{array}$} & \multirow{2}{*}{$\begin{array}{l}8.5 \\
(5)\end{array}$} & $\begin{array}{l}\text { Hardware/SW/UI } \\
\text { Improvement }\end{array}$ & $\begin{array}{l}2.0 \\
(13)\end{array}$ \\
\hline & & & & $\begin{array}{c}\text { Linking the Relevant } \\
\text { Systems }\end{array}$ & $\begin{array}{l}6.5 \\
(9)\end{array}$ \\
\hline & & $\begin{array}{l}\text { Security Issue } \\
\text { Improvement }\end{array}$ & $\begin{array}{l}6.6 \\
(6)\end{array}$ & $\begin{array}{l}\text { Public Authentication SW } \\
\text { Improvement }\end{array}$ & $\begin{array}{l}2.5 \\
(12)\end{array}$ \\
\hline
\end{tabular}




\begin{tabular}{|c|c|c|c|c|c|}
\hline & & & & $\begin{array}{l}\text { Personal Member } \\
\text { Information Encryption } \\
\text { Improvement }\end{array}$ & $\begin{array}{l}4.1 \\
(11)\end{array}$ \\
\hline \multirow{4}{*}{$\begin{array}{c}\text { G4B } \\
\text { Service } \\
\text { Improvement }\end{array}$} & \multirow{4}{*}{$\begin{array}{l}47.6 \\
(1)\end{array}$} & \multirow{2}{*}{$\begin{array}{l}\text { Online Administration } \\
\text { Service } \\
\text { Improvement/Expansion }\end{array}$} & \multirow{2}{*}{$\begin{array}{c}33.1 \\
(1)\end{array}$} & $\begin{array}{l}\text { Business Civil Affairs } \\
\text { Service and Support } \\
\text { Expansion }\end{array}$ & $\begin{array}{l}15.6 \\
(2)\end{array}$ \\
\hline & & & & $\begin{array}{l}\text { Provide Customized } \\
\text { Services for Business } \\
\text { Lifecycles }\end{array}$ & $\begin{array}{l}17.5 \\
(1)\end{array}$ \\
\hline & & \multirow{2}{*}{$\begin{array}{c}\text { Big Data-based } \\
\text { Business Extraction } \\
\text { Support }\end{array}$} & \multirow{2}{*}{$\begin{array}{l}14.5 \\
(4)\end{array}$} & $\begin{array}{l}\text { Provide Public/Private } \\
\text { DATA SET and Service } \\
\text { Linkage }\end{array}$ & $\begin{array}{l}6.7 \\
(7)\end{array}$ \\
\hline & & & & $\begin{array}{l}\text { Provide Consulting and } \\
\text { Information for Business } \\
\text { Extraction }\end{array}$ & $\begin{array}{l}7.8 \\
(5)\end{array}$ \\
\hline \multirow{5}{*}{$\begin{array}{c}\text { G4B } \\
\text { Policy } \\
\text { Improvement }\end{array}$} & \multirow{5}{*}{$\begin{array}{l}37.2 \\
(2)\end{array}$} & \multirow{2}{*}{$\begin{array}{l}\text { Legal policy } \\
\text { Improvement }\end{array}$} & \multirow{2}{*}{$\begin{array}{l}16.4 \\
(3)\end{array}$} & $\begin{array}{l}\text { Find Legal Evidence of } \\
\text { Linked/Integrated Service }\end{array}$ & $\begin{array}{l}9.2 \\
(3)\end{array}$ \\
\hline & & & & $\begin{array}{c}\text { Establish G4B Service } \\
\text { Promotion Strategy and } \\
\text { Roadmap }\end{array}$ & $\begin{array}{l}7.2 \\
(6)\end{array}$ \\
\hline & & \multirow{3}{*}{$\begin{array}{l}\text { Promoted System } \\
\text { Improvement }\end{array}$} & \multirow{3}{*}{$\begin{array}{c}20.9 \\
(2)\end{array}$} & $\begin{array}{l}\text { Construct Collaborating } \\
\text { Systems between } \\
\text { Departments }\end{array}$ & $\begin{array}{l}8.0 \\
(4)\end{array}$ \\
\hline & & & & $\begin{array}{l}\text { Establish Exclusive } \\
\text { Organizations }\end{array}$ & $\begin{array}{c}6.3 \\
(10)\end{array}$ \\
\hline & & & & $\begin{array}{l}\text { Prepare inter-ministerial } \\
\text { Adjustment Organizations }\end{array}$ & $\begin{array}{l}6.6 \\
(8)\end{array}$ \\
\hline total & $100 \%$ & total & $100 \%$ & total & $100 \%$ \\
\hline
\end{tabular}

\subsubsection{Comprehensive analysis of AHP results}

The levels of policy assignments for the G4B development was examined in terms of importance and urgency, and the results showed that "G4B policy improvement" was considered the most important assignment while "G4B service improvement" was considered to have the highest priority in terms of urgency. In large categories, the development of core services and the improvement of policy were considered to be more urgent and important than system improvement for the future spread of G4B and its constant usage.

In mid-level categories (indexes), "online administrative service improvement/expansion" was considered both important (28.7\%) and urgent (33.1\%), and "legal policy improvement" and "promotion system improvement" were considered the second most important and the third most urgent issues. The new G4B service deduction "big-data-based business deduction service support" was the fourth most important and urgent issue, and "system maintenance/repair" and "security issue improvement" showed lower priority in importance and urgency levels.

G4B development policy factors were examined based on professionals' reviews and it was found that the level and priority of importance and urgency did not exactly match up, although importance and urgency generally showed similar trends. We assume that these overlap because the system for G4B services was complete in 2012, and we are currently at an early stage in providing the service. 
Table 4.

\begin{tabular}{|c|c|c|c|c|c|c|c|c|}
\hline Major Class & $\begin{array}{c}\text { importanc } \\
\mathrm{e}\end{array}$ & $\begin{array}{c}\text { urgenc } \\
\mathrm{y}\end{array}$ & Mid Class & $\begin{array}{l}\text { importanc } \\
\mathrm{e}\end{array}$ & $\begin{array}{c}\text { urgenc } \\
\mathrm{y}\end{array}$ & Minor Class & $\begin{array}{c}\text { importanc } \\
\mathrm{e}\end{array}$ & $\begin{array}{c}\text { urgenc } \\
\mathrm{y}\end{array}$ \\
\hline \multirow{4}{*}{$\begin{array}{c}\text { G4B } \\
\text { System } \\
\text { Improvemen } \\
\mathrm{t}\end{array}$} & \multirow{4}{*}{$\begin{array}{c}13.3 \\
(3)\end{array}$} & \multirow{4}{*}{$\begin{array}{l}15.1 \\
(3)\end{array}$} & \multirow{2}{*}{$\begin{array}{l}\text { Maintain and Repair } \\
\text { System }\end{array}$} & \multirow{2}{*}{$\begin{array}{l}7.5 \\
(5)\end{array}$} & \multirow{2}{*}{$\begin{array}{l}8.5 \\
(5)\end{array}$} & $\begin{array}{l}\text { Hardware/SW/U } \\
\text { I Improvement }\end{array}$ & $\begin{array}{c}1.4 \\
(13)\end{array}$ & $\begin{array}{l}2.0 \\
(13)\end{array}$ \\
\hline & & & & & & $\begin{array}{l}\text { Linking the } \\
\text { Relevant } \\
\text { Systems }\end{array}$ & $\begin{array}{l}6.0 \\
(9)\end{array}$ & $\begin{array}{l}6.5 \\
(9)\end{array}$ \\
\hline & & & \multirow{2}{*}{$\begin{array}{l}\text { Security Issue } \\
\text { Improvement }\end{array}$} & \multirow{2}{*}{$\begin{array}{l}5.8 \\
(6)\end{array}$} & \multirow{2}{*}{$\begin{array}{l}6.6 \\
(6)\end{array}$} & $\begin{array}{c}\text { Public } \\
\text { Authentication } \\
\text { SW } \\
\text { Improvement }\end{array}$ & $\begin{array}{c}2.1 \\
(12)\end{array}$ & $\begin{array}{c}2.5 \\
(12)\end{array}$ \\
\hline & & & & & & $\begin{array}{c}\text { Personal } \\
\text { Member } \\
\text { Information } \\
\text { Encryption } \\
\text { Improvement }\end{array}$ & $\begin{array}{c}3.7 \\
(11)\end{array}$ & $\begin{array}{c}4.1 \\
(11)\end{array}$ \\
\hline \multirow{4}{*}{$\begin{array}{c}\text { G4B } \\
\text { Service } \\
\text { Improvemen } \\
\mathrm{t}\end{array}$} & \multirow{4}{*}{$\begin{array}{l}42.8 \\
(2)\end{array}$} & \multirow{4}{*}{$\begin{array}{l}47.6 \\
(1)\end{array}$} & \multirow{2}{*}{$\begin{array}{l}\text { Online Administration } \\
\text { Service } \\
\text { Improvement/Expansio } \\
n\end{array}$} & \multirow{2}{*}{$\begin{array}{c}28.7 \\
(1)\end{array}$} & \multirow{2}{*}{$\begin{array}{c}33.1 \\
(1)\end{array}$} & $\begin{array}{l}\text { Business Civil } \\
\text { Affairs Service } \\
\text { and Support } \\
\text { Expansion }\end{array}$ & $\begin{array}{l}10.3 \\
(3)\end{array}$ & $\begin{array}{l}15.6 \\
(2)\end{array}$ \\
\hline & & & & & & $\begin{array}{l}\text { Provide } \\
\text { Customized } \\
\text { Services for } \\
\text { Business } \\
\text { Lifecycles }\end{array}$ & $\begin{array}{l}18.4 \\
(1)\end{array}$ & $\begin{array}{l}17.5 \\
(1)\end{array}$ \\
\hline & & & \multirow{2}{*}{$\begin{array}{l}\text { Big Data-based } \\
\text { Business Extraction } \\
\text { Support }\end{array}$} & \multirow{2}{*}{$\begin{array}{l}14.1 \\
(4)\end{array}$} & \multirow{2}{*}{$\begin{array}{l}14.5 \\
(4)\end{array}$} & $\begin{array}{c}\text { Provide } \\
\text { Public/Private } \\
\text { DATA SET and } \\
\text { Service Linkage }\end{array}$ & $\begin{array}{l}6.5 \\
(8)\end{array}$ & $\begin{array}{l}6.7 \\
(7)\end{array}$ \\
\hline & & & & & & $\begin{array}{c}\text { Provide } \\
\text { Consulting and } \\
\text { Information for } \\
\text { Business } \\
\text { Extraction }\end{array}$ & $\begin{array}{l}7.6 \\
(5)\end{array}$ & $\begin{array}{l}7.8 \\
(5)\end{array}$ \\
\hline \multirow{5}{*}{$\begin{array}{c}\text { G4B } \\
\text { Policy } \\
\text { Improvemen } \\
\mathrm{t}\end{array}$} & \multirow{5}{*}{$\begin{array}{c}43.9 \\
(1)\end{array}$} & \multirow{5}{*}{$\begin{array}{l}37.2 \\
(2)\end{array}$} & \multirow{2}{*}{$\begin{array}{l}\text { Legal } \\
\text { policyImprovement }\end{array}$} & \multirow{2}{*}{$\begin{array}{l}23.6 \\
(2)\end{array}$} & \multirow{2}{*}{$\begin{array}{l}16.4 \\
(3)\end{array}$} & $\begin{array}{c}\text { Find Legal } \\
\text { Evidence of } \\
\text { Linked/Integrate } \\
\text { d Service }\end{array}$ & $\begin{array}{l}15.3 \\
(2)\end{array}$ & $\begin{array}{l}9.2 \\
(3)\end{array}$ \\
\hline & & & & & & $\begin{array}{l}\text { Establish G4B } \\
\text { Service } \\
\text { Promotion } \\
\text { Strategy and } \\
\text { Roadmap }\end{array}$ & $\begin{array}{l}8.3 \\
(4)\end{array}$ & $\begin{array}{l}7.2 \\
(6)\end{array}$ \\
\hline & & & \multirow{3}{*}{$\begin{array}{l}\text { Promoted System } \\
\text { Improvement }\end{array}$} & \multirow{3}{*}{$\begin{array}{l}20.3 \\
(3)\end{array}$} & \multirow{3}{*}{$\begin{array}{l}20.9 \\
(2)\end{array}$} & $\begin{array}{c}\text { Construct } \\
\text { Collaborating } \\
\text { Systems between } \\
\text { Departments }\end{array}$ & $6.9(7)$ & $\begin{array}{l}8.0 \\
(4)\end{array}$ \\
\hline & & & & & & $\begin{array}{l}\text { Establish } \\
\text { Exclusive } \\
\text { Organizations }\end{array}$ & $\begin{array}{c}6.0 \\
(10)\end{array}$ & $\begin{array}{c}6.3 \\
(10)\end{array}$ \\
\hline & & & & & & $\begin{array}{l}\text { Prepare inter- } \\
\text { ministerial } \\
\text { Adjustment } \\
\text { Organizations }\end{array}$ & $\begin{array}{l}7.4 \\
(6)\end{array}$ & $\begin{array}{l}6.6 \\
(8)\end{array}$ \\
\hline
\end{tabular}

\subsection{Policy Proposal for G4B Development}

Based on the AHP analysis result, the main assignments for G4B development will be divided in terms of service and system. 


\subsubsection{Services That Requires Improvement}

First, contents that reflect the demand of online business support for service consumers must be enhanced. In particular, aside from current services that focus only on start-up business support and civil applications, online services pertaining to the entire cycle of business, such as opening/growing/closing the business, exportation, fund support, and recruiting, must be expanded.

Second, service methods such as simplified menus, intuitive UI, various connection paths, and enhanced community features between users must be improved in order to increase the accessibility and usability of G4B services.

Third, for the mid-long-term plan, it is necessary to introduce new services that can transform creative ideas into businesses and actively grow such businesses. For example, for businesses based in a town with a creative economy that is currently managed by the Ministry of Science, ICT, and Future Planning, it will be possible to use G4B to supply online services that provide information for business start-ups and customized fund support for businesses' after their incubation period. This can lead to a Korean model that can pioneer the idea of seamlessly opening, managing, and supporting businesses.

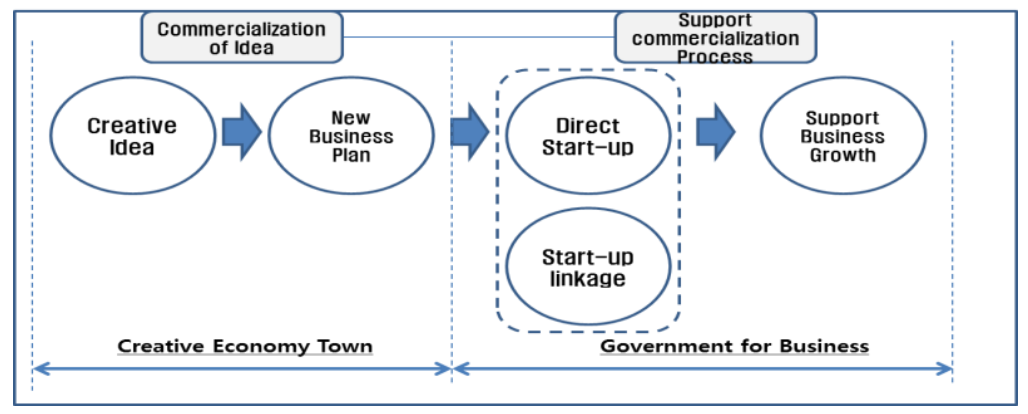

Figure 1.

Fourth, a tighter link between similar businesses that provide online business support is vital. It is necessary to actively consider strengthen connections between the services offered by various government departments like G4B of MSIP, SMBA related service sites, and minwon24 in MOGAHA, which is helpful to organically provide online business support services, and integrate them in the mid and long term.

Table 5.

\begin{tabular}{|c|c|c|c|c|c|c|c|c|c|c|c|}
\hline \multirow{2}{*}{\multicolumn{2}{|c|}{ Service }} & \multirow{3}{*}{$\begin{array}{c}\text { Start } \\
\text {-up } \\
0\end{array}$} & \multicolumn{3}{|c|}{ Business Management } & \multirow{3}{*}{$\begin{array}{c}\text { Change } \\
\text { of } \\
\text { Busine } \\
\text { ss }\end{array}$} & \multicolumn{2}{|c|}{ Business closure } & \multicolumn{3}{|c|}{ Others } \\
\hline & & & \multirow{2}{*}{$\begin{array}{c}\text { Civil } \\
\text { Affairs } \\
\text { Services }\end{array}$} & \multirow{2}{*}{$\begin{array}{c}\text { Authentication } \\
\text { () }\end{array}$} & \multirow{2}{*}{$\begin{array}{c}\text { Support } \\
\Delta\end{array}$} & & \multirow{2}{*}{$\begin{array}{c}\text { closure } \\
\end{array}$} & \multirow{2}{*}{$\begin{array}{c}\text { rehabilitation } \\
\mathrm{X}\end{array}$} & \multirow{2}{*}{$\begin{array}{c}\text { education } \\
\Delta\end{array}$} & \multirow{2}{*}{$\begin{array}{c}\text { consulting } \\
\Delta\end{array}$} & \multirow{2}{*}{$\begin{array}{c}\text { funding } \\
\end{array}$} \\
\hline \multirow{2}{*}{$\begin{array}{l}\text { G4B in } \\
\text { MSIP }\end{array}$} & $\begin{array}{c}\text { Informati } \\
\text { on } \\
\text { Service }\end{array}$ & & & & & & & & & & \\
\hline & $\begin{array}{l}\text { Online } \\
\text { Applicati } \\
\text { on }\end{array}$ & O & $\bigcirc$ & (C) & $\mathrm{X}$ & (a) & $\mathrm{X}$ & $\mathrm{X}$ & $\Delta$ & $\Delta$ & $\mathrm{X}$ \\
\hline \multirow{2}{*}{$\begin{array}{l}\text { Bizinfo in } \\
\text { SMBA }\end{array}$} & $\begin{array}{c}\text { Informati } \\
\text { on } \\
\text { Service }\end{array}$ & $\bigcirc$ & $\bigcirc$ & $\bigcirc$ & $\bigcirc$ & $\mathrm{X}$ & $\mathrm{X}$ & $\Delta$ & (C) & (C) & (C) \\
\hline & $\begin{array}{c}\text { Online } \\
\text { Applicati } \\
\text { on }\end{array}$ & $\bigcirc$ & $\bigcirc$ & $\bigcirc$ & $\mathrm{X}$ & $\mathrm{X}$ & $X$ & $\Delta$ & (C) & (C) & $\mathrm{X}$ \\
\hline $\begin{array}{l}\text { Minwon } \\
\text { in } \\
\text { MOGAH }\end{array}$ & $\begin{array}{c}\text { Informati } \\
\text { on } \\
\text { Service }\end{array}$ & $\mathrm{X}$ & $\bigcirc$ & $\mathrm{X}$ & $\mathrm{X}$ & $\mathrm{X}$ & $\mathrm{X}$ & $\mathrm{X}$ & $\mathrm{X}$ & $X$ & $\mathrm{X}$ \\
\hline
\end{tabular}




\begin{tabular}{c|c|c|c|c|c|c|c|c|c|c|c}
\hline A & $\begin{array}{c}\text { Online } \\
\text { Applicati } \\
\text { on }\end{array}$ & $\mathrm{X}$ & $\mathrm{O}$ & $\mathrm{X}$ & $\mathrm{X}$ & $\mathrm{X}$ & $\mathrm{X}$ & $\mathrm{X}$ & $\mathrm{X}$ & $\mathrm{X}$ & $\mathrm{X}$ \\
\hline
\end{tabular}

\subsubsection{Improvements Regarding Legal Policies}

First, creating an internal department for G4B will be considered to be a priority. Currently, the Green Integration Department of SW Integration Department in IT $\cdot \mathrm{SW}$ Integration Group is in charge of G4B operations in NIPA, and this department only consists of two employees. If G4B develops to become a multi-departmental business, the current amount of work-related tasks, such as linking businesses between relevant departments, will increase and, therefore, the current number of employees must be expanded to create a team-level organization.

Second, in the Ministry of Science, ICT, and Future Planning, G4B-related tasks are categorized as software integrations and primary departmental tasks under the software policy of Budget and Treasury information and communication. It may be difficult to lead businesses and deduct practical discussion items at an official level when G4B develops into an inter-ministerial collaborative business. Therefore, it will be necessary to enhance the internal promotion system and change the positions of G4B group members (action officer $\rightarrow$ deputy director) in the Ministry of Science, ICT, and Future Planning and internally review whether G4B is a SW-related operation.

Third, the promotion system must be repaired to construct governmental departments for online business support services. In particular, for cooperation between department consultative groups between related departments such as the Ministry of Science, ICT, and Future Planning; Ministry of Trade, Industry, and Energy; Ministry of Government Administration and Home Affairs; Small and Medium Business Administration; an interministerial and private sector cooperation consultative group; and a senior adjustment organization should be prepared, and the expansion of a memorandum of understanding between departments should be specifically discussed. A roadmap consisting of basic plans (ICT strategies) and practical plans (each department) must be recorded, and the relevant improvement of legislation and preparation of new laws such as integrated G4Brelated special laws must be established.

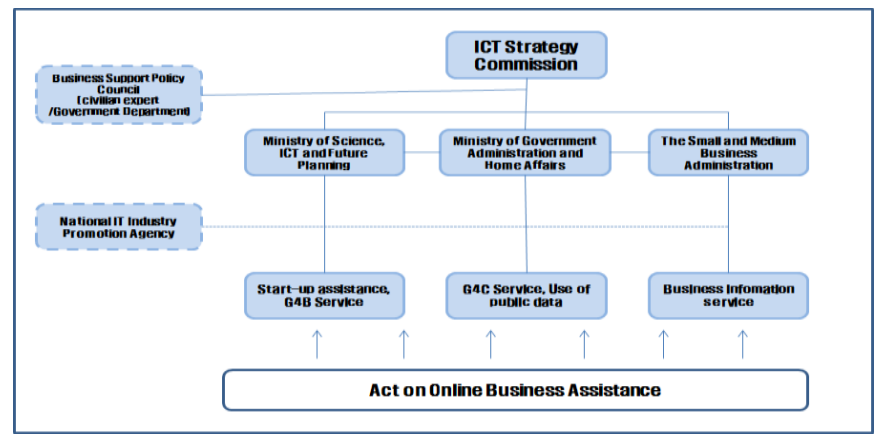

Figure 2.

\section{References}

[1] J. G. Kim and E.-J. Kim, "Creative Industries' Export and Internationalization Strategies of Selected Countries and Their Policy Implications", KIEP Studies in Comprehensive Regional Strategies,vol. 13,no. 15,(2013), pp. 13-20.

[2] "Ministry of Science", ICT and Future Planning, Creative Economy Action Plan,(2013).

[3] "Ministry of Government Administration and Home Affairs", Government 3.0 Basic Plan,(2013).

[4] "National Information Agency", Thecreative economy paradigm and ICT policy direction,(2013).

[5] B.-H.Meng, "Effects of the government e-procurement project", The conference of The KoreanAssociation for Policy Science,(2002).

[6] J. W. Jung, "The information policy of Lee Myung-bak government: focused on e-Government project", the 2009 conference of The KoreanAssociation for Policy Analysis and Evaluation,(2009). 
[7] T. L. Saaty, "The Analytic Hierarchy Process: Planning, Priority Setting, Resource Allocation", New York: McGraw-Hill Book Co.,(1980).

[8] K. T. Cho, Y. K. Choand H. S. Kang, "Analytic Hierarchy Process", Seoul: Dong Hyun Publisher,(2005).

[9] K. K. Ko andH. Y. Ha, "Meta-Analysis of the Utilization of Analytic Hierarchy Process for Policy Studies in Korea," Journal of the Korean Association for Policy Studies, vol. 17, no.1,(2008), pp.287313.

[10] T. Y. Lee, "The understanding of Analytic Hierarchy Process", the Board of Audit and Inspection, a quarterly Audit and Inspection,(2006).

[11] S. J. Cho andS. J. Jo, "The Evaluation of the Customer Satisfaction Rate for G2B Service," Journal of Korean Society and Public Administration, vol. 15, no.3,(2004), pp. 229-256.

[12] P. K. Hong, "The exploratory study on the measurement of e-government's efficiency", The Korean Association for Regional Information Society, vol.10, no. 1,(2007),pp. 165-191.

[13] C. S. Jung,e-Government, Seoul: Seoul Economy \& administration Publisher,(2015).

[14] J. M. Kim, "A Study on the Status and Improvement Direction of Korea SMEs Policy Information System," Journal of the Korea Society of IT Services, vol. 7, no.4,(2008), pp.61-86.

[15] S. I. Han, "Factors Influencing the Intention of using G4C(Government for Citizen)," Journal of CEO and Management Studies, vol. 13, no.1,(2010), pp. 213-240.

[16] C. S. ChungandW. J. Sung, "Research on the Development Direction of Online Business Support System", Workshop of Science \& Engineering Research Support Society,(2015) August.

[17] K. S. Kim, "Smart Government and Cloud data center", National Computing \& Information Agency,(2011).

[18] T. J. Yang, B. R.Seo, W. S. Jang, C. Y. Hong, M. H. Lee andY. S. Kim, “e-government promotion plan though the G4C case", the 2004 conference of The Korean Association for Public Administration, (2004).

[19] "Korea Information Society Development Institute", The Analysis of the Korean Administration informatization, (2008).

[20] C. S. Chung, "A Case Study on Business Process Innovation in a Window for Civil Petitions trough Public Information Sharing: Focus on Policy Advice for Business Process Reengineering," Entrue Journal of Information Technology, vol. 7,no.1,(2008), pp.63-77.

[21] C. H. Jung andC. J. Lee, "The Evaluations of Enterprise Supporting Portal in Local Authorities Using AHP Method: Focused on D Public Organization”, The Korea Contents Association Institute,(2010), pp.450-452.

[22] http://www.msip.go.kr/web/main/main.do.

[23] https://www.creativekorea.or.kr/.

[24] http://www.g4b.go.kr/.

[25] http://www.smba.go.kr/kr/index.do.

[26] http://www.bizinfo.go.kr/.

[27] http://www.mogaha.go.kr/.

[28] http://www.minwon.go.kr/.

[29] http://www.nia.or.kr/.

\section{Authors}

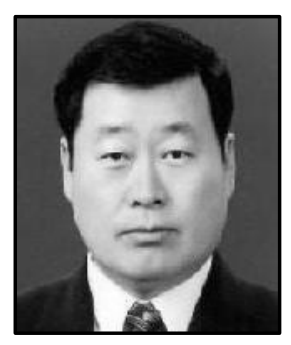

\section{ChoongSik Chung}

. Dept. of Public Administration, Kyungsung University

. Professor

. e-Government, Information Policy

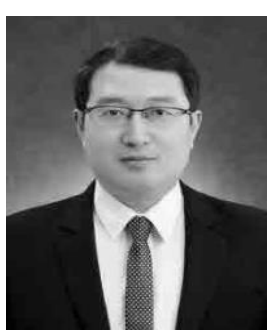

\section{Wookjoon Sung}

. Graduate School of Public Policy \& Infomation Tech., Seoul National University of Science \& Technology

. Assistant Professor

. ICT Policy, e-Government, Policy Analysis \& Evaluation 